\title{
MEDICAL TREATMENT OF ULCERATIVE COLITIS
}

\author{
By S. C. Truelove, M.A., M.D.(Саmb.), M.R.C.P. \\ Nuffield Department of Clinical Medicine, University of Oxford
}

There is no cure for ulcerative colitis and a patient who has once suffered from an attack is likely to have recurrent attacks. Fortunately, some patients have many years of perfect health in between these attacks, while a few have a single attack only and never have another one. At the other extreme, some patients suffer from continuous symptoms without ever becoming entirely free of them, although such cases are less common now than previously. For the usual type of case which pursues an intermittent course medical treatment resolves itself into the most efficient treatment of each attack as it arises. Ulcerative colitis varies greatly in severity from one patient to another and in the same patient from time to time. It is therefore convenient to discuss medical treatment according to the severity of the attack which is to be treated.

\section{Medical Management of a Severe Attack}

By a severe attack is implied an illness in which a profuse bloody diarrhoea is accompanied by gross constitutional disturbances, such as fever, tachycardia, a falling haemoglobin level, rapid loss of weight, muscular weakness and general malaise. Such a severe attack often supervenes upon milder symptoms which have been persisting for weeks or months, but in some patients, especially in the case of first attacks, the illness may become severe with alarming rapidity, so that in the course of a few days an erstwhile healthy person becomes gravely ill. Such a patient should be admitted without delay to a first-class hospital and the general medical measures set out below be instituted immediately. As soon as the diagnosis is made, which is usually not difficult in these severe attacks, the other measures which are designed to shorten the attack should be brought into play.

\section{General Medical Measures}

I. Correction of dehydration and electrolyte depletion. In any case of profuse diarrhoea there is heavy loss, not only of water, but of electrolytes. In particular, the loss of potassium is usually heavy and the patient may be rendered gravely ill on this score alone. If possible, correction should be carried out by the oral administration of appropriate replacement fluids, but if there is vomiting or the electrolyte disturbance is very severe, parenteral administration will be necessary. Once corrected, regular observations should be made on the electrolytes while the illness is in a severe phase, so that appropriate corrective measures can be instituted whenever necessary.

2. Blood transfusion repeated as often as is necessary should be carried out to maintain tho haemoglobin at a high level. By this means the blood which is being lost per rectum is replaced without the body having to call upon its own protein stores to make good the loss. As nitrogen loss from the bowel is very heavy in severe ulcerative colitis, this consideration is of great importance if rapid wasting is to be avoided.

3. A high-calorie intake, preferably with a large amount of protein, should be instituted. This is often easier said than done, because, when the attack is severe, vomiting and anorexia are common. In such cases the use of a gastric drip at night under the cover of heavy sedation may help to get a reasonable number of calories into the body.

4. Vitamin supplements should be given, because, although there is no evidence that ulcerative colitis is due to lack of any known vitamin, deficiencies can occur because of the disease. B-complex should be given parenterally if the attack is severe, especially if antibiotics are being used. As well as the more usual vitamins, folic acid and $B_{12}$ are possibly worth giving if a severe attack continues for more than a week or two. There is no certain evidence that they do any good, but their use is harmless.

\section{Specific Agents Used in the Hope of Cutting Short the Attack}

I. ACTH and corticosteroids used systemically. 
2. Water-soluble compounds of hydrocortisone and prednisolone used locally in the bowel.

3. Salazopyrin.

(A discussion of the use of these specific agents will follow later )

\section{Accessory Measurès}

I. Anticholinergics can be given to minimize the diarrhoea and to ease abdominal pain. A suitable preparation is probanthine, $\mathrm{I} 5$ to $30 \mathrm{mg}$. four times a day by mouth. Larger doses should be avoided, as they are likely to give rise to abdominal distention, anorexia and vomiting.

2. Heavy sedation at night to ensure sleep and also to help quieten the bowel.

3. Penicillin and sulphonamides are worth using in conjunction with systemic corticoid therapy, especially when the patient is febrile. The combined use of penicillin and streptomycin by intramuscular injection is also a good way to combat the risk of pyogenic complications in severe cases. Broad-spectrum antibiotics can be given for a few days if fever persists, but it is best to choose one which can be given parenterally and to keep to a short course of treatment.

\section{Measures to be Avoided}

1. Oral iron preparations. During an attack of ulcerative colitis the patient is losing blood and this should be replaced by transfusion with whole blood. Iron by mouth is irritating to the gastrointestinal tract and often causes an exacerbation of the symptoms of ulcerative colitis. There is also evidence that iron is poorly utilized during severe ulcerative colitis, so from all points of view iron therapy should be avoided.

2. Broad-spectrum antibiotics are best avoided as a general rule and especially when given by mouth. It is well known that they are themselves liable to create a dangerous entero-colitis. The risk of this is small in a patient with normal bowel habit if the antibiotic is stopped at the first sign of any looseness of the bowel, but in a patient with ulcerative colitis such a premonitory sign is plainly impossible to perceive. In general, therefore, it is wiser to avoid their oral use and to employ them for short courses of a few days by a parenteral route in selected patients only.

3. Opiates are best avoided. The habit of giving large quantities of tincture of opium in an attempt to check the diarrhoea is to be condemned. Opiates have little effect upon the diarrhoea in a severe case unless given in such large quantities that the patient is fuddled by them. There is also the risk of drug addiction.
Medical Management of Moderate and Mild Attacks

These attacks need to be treated promptly, because, if neglected, they may become severe. Moderate attacks often need admission to hospital. Treatment follows the same lines as for a severe attack, but can proceed at a more leisurely tempo and not all the measures may be necessary.

Attacks which are relatively mild can be treated while the patient remains ambulant. At present my first choice would be to use local treatment with hydrocortisone. If this fails to bring about remission in two weeks, prednisone, $5 \mathrm{mg}$. q.d.s., can be given in addition. If this combination fails, salazopyrin, I g. q.d.s., can be tried, either alone or in combination with local hydrocortisone or with a systemic corticoid. The few attacks which fail to respond to one or other of these combinations should be put on to ACTH, which is best given in the form of a gel or colloidal suspension.

\section{Discussion of Agents Used to Cut Short an Attack of Ulcerative Colitis}

At present some type of corticoid therapy or the use of salazopyrin seem to be the best measures available for cutting short an attack.

The use of corticoids in this disease has been the subject of controversy and the argument has not yet died down. Shortly after the introduction of cortisone for rheumatoid arthritis, various workers tried this agent or ACTH in ulcerative colitis. Some found that corticoid therapy would frequently produce rapid symptomatic relief, while others found an apparently increased incidence of dangerous complications. For example, Tulin et al. (1952) treated 17 patients with ACTH and had three colonic perforations and they judged that this agent was too dangerous to use in an acute attack. Even among those who favoured the use of these agents there was disagreement as to the type of case in which they should be used, some physicians advocating their use in acute attacks, while others thought they should be reserved for mild ones. To a large extent these issues were resolved by a large-scale controlled trial carried out by a number of physicians working to a common plan. This showed that at every level of severity the cortisone-treated patients fared better than those in the control group (Truelove and Witts, 1954, 1955). However, oral cortisone emerged as a far from perfect treatment of the actual attack and the liability to later attacks was little affected by this treatment. Moreover, some surgeons still consider that the use of cortisone may prejudice the chance of surgery if this becomes necessary, especially if continued for more than a week or two in the absence of a favourable clinical response (Brooke, 1956). An important practical 
point is that, if surgery becomes necessary in a patient who has been on systemic corticoid therapy for more than a few days, ample doses of hydrocortisone should be given during the operative period and the next few days because the patient's own adrenals may not respond adequately to the stress of surgery. Surgeons are not alone in distrusting corticoids and the case against them can be found in articles by distinguished physicians, such as Bargen (1955) and Bockus (1958). However, in centres where corticoid therapy has been greatly used, the results for the disease have shown a marked improvement and the bogy of increased complications does not seem to have materialized (Maltby et al., 1956; Kirsner and Palmer, 1954; Kirsner, Ricks and Palmer, I957; Kirsner, Sklar and Palmer, 1957).

There has been some dispute as to which is the best form of corticoid therapy. A recent controlled trial has shown that ACTH in a daily dose of 80 units of the gel has much the same effect as oral cortisone in a dose of $50 \mathrm{mg}$. q.d.s. as far as first attacks of the disease are concerned, but that ACTH produces twice as many clinical remissions in relapses of established disease (Truelove and Witts, 1959). However, ACTH carries the disadvantages of a greater liability to hypercortisonism, including mental disturbances, to peptic ulcer complications and to moniliasis, so in general it seems best to hold it in reserve to be used if a prompt response is not obtained with cortisone or one of its analogues. The newer oral compounds, such as hydrocortisone, prednisone, prednisolone and triamcinolone, have been thought to be intermediate in potency between cortisone and ACTH (Kirsner, Ricks and Palmer, 1957; Fierst et al., 1957), but exact comparisons are not yet available and each would be a major undertaking.

An alternative method of using corticoids is to employ them locally in the colon in a manner analogous to the use of a hydrocortisone lotion in treating skin diseases or of eye-drops for ocular diseases. Hydrocortisone is superior to cortisone for local use and a water-soluble compound of hydrocortisone, such as the hemisuccinate, is to be preferred to the free alcohol. The use of hydrocortisone topically by means of a nightly rectal drip brings about speedy remission of symptoms in a large proportion of mild and moderate attacks (Truelove, 1956, 1957). Recently two independent studies made by the 'double blind ' technique have confirmed the validity of this conclusion (Truelove, 1958; Watkinson, 1958). There still remain some problems to be settled with respect to this form of local therapy. One concerns the agent to be used. Hydrocortisone hemisuccinate sodium has proved generally satisfactory, but recent experience with another water-soluble compound; prednisolone
2I-phosphate, shows this to be at least as good and possibly better. Another is whether local treatment is superior to systemic use of corticoids. Local treatment usually acts much more quickly $\stackrel{\varrho}{.}$ and side-effects of therapy are absent. In addition, $\overrightarrow{\bar{F}}$ a study as yet incomplete suggests that the proportion of favourable responses is decidedly higher with local treatment. However, the most efficient way of using corticoid therapy may prove to be the combined use of local treatment and a systemic corticoid, the systemic use being abandoned as soon as a good symptomatic response is obtained; this possibility is also being explored at present. Yet another problem is how best to use local therapy in the treatment of very severe attacks when diarrhoea is profuse. At present the most important aid is the giving of intravenous probanthine, 15 to $30 \mathrm{mg}$. shortly before starting the rectal drip. Another is to give hydrocortisone hemisuccinate intravenously, roo mg. b.d. for a few days, while the local treatment is in process of $\mathrm{N}$ taking effect; the few patients with severe attacks $ᄋ$ who have so far been treated on this particular combined regime have all shown a rapid, favourable response, but further experience will be necessary to decide whether it is the best combination.

Salazopyrin has been extensively used in Scane $\vec{\varphi}$ dinavia and America, but was not available in this country until about three years ago and has not ye enjoyed any widespread vogue here. It is a chemicat combination of sulphapyridine with salicylic acid, with the official name of salicylazosulphapyridine, but known as salazopyrin in this country and as azopyrin or asulfidine in the U.S.A. It was introduced in 1940 by Svartz, who has written a number of articles on its use (Svartz, 1942, 1948, 1954, 1956). Another Scandinavian adherent is Lagencrantz (1949, 1955), who has found it beneficial in children with the disease. Some physicians in America are enthusiastic about its virtues. For example, Bargen (1944) has written that " the most valuable drug that has been introduced for the treatment of ulcerative colitis in the years that $I$ have been interested in the problem is salicylazosulfapyridine.' It must, however, be pointed out that no properly controlled study has ever been made of this agent. The nearest approach to one is the study by Morrison (1952, 1953), in which he N compared 60 patients treated with salazopyrin with $N$ 60 patients treated in the preceding three years by $N$ other methods and showed that the salazopyrin- $\omega$ treated patients fared better. There are two reasons for viewing his results with a certain scepticism. Firstly, it is dangerous to compare two groups of patients seen in different time periods and then to attribute any improvement to the use of a single therapy unless the results show a startling improve- 
ment. Secondly, cortisone came into use at the same time as Morrison was treating his patients with salazopyrin, and Morrison himself implies that some of these patients received cortisone in addition to salazopyrin; as cortisone certainly improves the chance of remission, some of the beneficial effects attributed by Morrison to salazopyrin may have been due in reality to cortisone.

In brief, the conclusion that salazopyrin is very beneficial in ulcerative colitis rests upon flimsyfoundations if we adopt strict scientific criteria. However, my own experience of treating about 50 patients with it supports the view that it is of value, although I can bring forward no evidence other than my clinical impressions. It has seemed to me particularly valuable in patients with mild, continuous symptoms and it has the virtue in such cases of being relatively safe to use for quite long periods of time. It is prescribed as tablets of $0.5 \mathrm{~g}$., the full dose for a severe attack of ulcerative colitis being 2 to 3 g. q.d.s., this dose being reduced after a week or so. For milder cases, I g. q.d.s. appears to be sufficient and for maintenance treatment a still smaller dose can be used. A considerable number of patients experience nausea or vomiting with the larger doses, so that treatment may need to be temporarily suspended or given at a lower level. Fever and drug rashes have been described and neutropenia exists as a dangerous threat, although I have not seen these complications personally. In Oxford we have found the main complication (other than the gastric symptoms) to be Heinz-body anaemia, of which we have now had six cases among patients treated with this agent, the first four having already been reported (Spriggs et al., 1958). This is not a serious objection to the use of the drug once the risk is appreciated and arrangements made for regular blood examination with this specific point in mind. The drug can be used in combination with cortisone and its analogues and this combination has seemed to me useful. In such combined treatment gross water retention occasionally occurs with marked oedema; salazopyrin certainly plays a large part in this effect, because patients have lost their oedema on stopping the salazopyrin, even though they have continued on corticoid therapy.

Many other agents have been used from time to time as specific therapy in ulcerative colitis. Examples are: anti-thyroid drugs, intestinal extracts, massive doses of vitamin $\mathrm{B}_{12}$, large doses of ascorbic acid, apple extracts, crude liver extracts, vitamin $P$, salicylates, isoniazid, starch and opium retention enemata. I know of no convincing evidence that any of these have any major effect upon the course of the disease. However, it is sometimes convenient to use one or other of them when a patient continues to get symptoms, as it

leads to an optimistic feeling on the part of the patient (and of the physician) that something is being done. An essential in such circumstances is to choose a safe agent so that, even if one does no good, at least one does no harm. However, during recent years I have personally found no need for this type of approach, although I used it earlier.

\section{Psychotherapy}

There is a widespread belief that ulcerative colitis is a psychosomatic disorder and there is an extensive literature on the subject. The importance of this view as far as treatment is concerned is that some workers have decided that the corollary is that treatment should be primarily psychological. In my own view, psychotherapy has little part to play at the height of an acute attack and its employment should never imply that the general principles of medical management mentioned earlier can be abandoned. In a less acute phase any emotional difficulties should be dealt with if possible, but I consider that few patients need formal psychotherapy. Some physicians believe that treatment should be primarily psychological, but that it is best carried out by a physician rather than by a professional psychiatrist. A protagonist of this approach is Paulley (1956), whose article is particularly valuable because it gives a clear account of the method of superficial psychotherapy which he uses.

\section{Treatment of Complications}

All the foregoing has dealt with uncomplicated disease, but ulcerative colitis is liable to show many complications. Some of the more common ones are set out in tabular form below, together with my views on the way they should usually be treated.

Local Complications

(a) Usually occurring in acute attack:

Ischio-rectal abscess. Fistula formation (fistula-in-ano, rectovaginal fistula)

Perforation of colon. Massive haemorrhage from bowel.

(b) Usually occurring as a result of chronic disease : Polyposis.

Fibrous stricture. Carcinoma.

Remote Complications Various skin lesions.

Arthritis (often associated with skin lesions,

\section{Usual Treatment}

Surgical drainage. Conservative surgery if possible. Otherwise colectomy.

Colectomy.

Blood transfusion. Occasionally colectomy may be necessary.

Keep under regular observation.

Colectomy.

Colectomy.

Treat the colitis. Give ample vitamins.

Treat the colitis. Corticoids usually stop the 
especially erythema nodosum).

Eye complications, especially conjunctivitis and iritis.

Aphthous ulceration of mouth.

Moniliasis of mouth and throat. arthritis. Occasionally a reason for colectomy.

Treat the colitis. Hydrocortisone eye-drops often indicated but eye-specialist's opinion should be sought.

Treat the colitis. Mouth tablets of hydrocortisone hemisuccinate sodium (Truelove and MorrisOwen, 1958).

Stop any broad-spectrum antibiotic being used. Stop any systemic corticoid being used. Ample vitamins. Nystatin tablets to suck and/or for systemic use.

\section{Long-term Measures}

In my own experience, the vast majority of attacks of ulcerative colitis can be brought under control by medical treatment along the lines already set out. The great problem is to prevent attacks recurring and at present there is no satisfactory solution. Attacks can probably be prevented by maintaining corticoid therapy at a full dose, but this in itself carries a number of risks and does not appear to be desirable. As far as using a small dose is concerned, a recent blind trial comparing $25 \mathrm{mg}$. b.d. of cortisone with inert tablets has shown no beneficial effect whatsoever from the use of cortisone (Truelove and Witts, I959). In a similar disappointing way, the use of local hydrocortisone at weekends only has failed to influence the relapse rate (Truelove, I958). ACTH has been advocated for long-term maintenance therapy, but one hesitates to advocate extensive use of a therapy carrying complications in its train in the absence of a properly controlled therapeutic trial. A small dose of salazopyrin can be used for a long time with negligible risks and often appears to be helpful, especially in patients with mild but chronic symptoms.

At present the best solution seems to be for these patients to keep in touch with a physician interested in the disease so that recurrent attacks can be treated in the earliest stages. If this is done, most of them can be satisfactorily controlled on an out-patient basis without the patient's life being seriously disrupted.

\section{Results}

Although our present medical measures are far $\frac{2}{\mathbb{D}}$ advance on previous therapy. Certainly in Oxford results for the disease have shown conspicuous $\stackrel{5}{\rightarrow}$ improvement during the last few years, as can be illustrated by a few figures. In a study of the hospital experience during the period 1938-49, $\frac{\overline{\bar{c}}}{\bar{\sigma}}$ Rice-Oxley and Truelove (1950) found that first $\stackrel{\mathbb{\Omega}}{\varrho}$ attacks were frequently severe and that about one in five patients died in hospital during this first attack. Taking recent experience, the last death $\circ$ in a first attack took place in r954. Only two of the $\overrightarrow{\vec{\omega}}$ first attacks have been treated surgically in this $\stackrel{\omega}{\sigma}$ period, so that medical treatment can be given ${ }_{0}$ credit for the improvement. It remains to be seen 3 . whether the long-term results for the disease will is show an equal improvement, but there are encouraging signs that this will prove to be so.

\section{BIBLIOGRAPHY}

BARGEN, J. A. (1955), Sth. med. F., 48, 192.

BOCKUS, H. L. (1958) 'Modern Trends in Gastroenterology, Second Series. Edited by F. Avery Jones. Butterworth London, p. $3 \mathrm{II}$.

BROOKE, B. N. (1956), Lancet, ii, 1175.

FIERST, S. M., ROBINSON, E., LANGSAM, A., WERNER, A., and INGEGNO, A. P. (1957), f. Amer. med. Ass., 163, 1444.

KIRSNER, J. B., and PALMER, W. L. (1954), Ann. intern. Med.

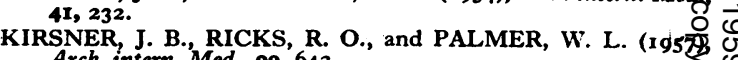
Arch. intern. Med., 99, 642.

KIRSNER, J. B., SKLAR, M., and PALMER, W. L. (195 Amer. ' F. Med., 22, 264.'

LAGENCRANTZ, R. (1949), Acta paediat., Stockh., suppl. No. 75, p. 89.

LAGENCRANTZ, R. (1955), Ibid., 44, 302.

MALTBY, E. J., DICKSON, R. C., and O'SULLIVAN, P. M. (1956), Canad. med. Ass. Э., 74, 4.

MORRISON, L. M. (1952), Gastroenterology, 21, 133.

MORRISON, L. M. (1953), F. Amer. med. Ass., 15I, 366.

PAULLEY, J. W. (1956), Lancet, ii, 215.

RICE-OXLEY, J. M., and TRUELOVE, S. (1950), Ibid., i, 663.

SPRIGGS, A. I., SMITH, R. S., GRIFFITH, H., and TRUELOVE, S. C. (1958), Ibid., i,' 1039.

SVARTZ, N. (1942), Acta med. scand., 110, 577.

SVARTZ, N. (1948), Ibid., suppl. No. 206, p. 465.

SVARTZ, N. (1954), Gastroenterology, 26, 26.

SVARTZ, N. (1956), Gastroenterologia (Basel), 86, 683.

TRUELOVE, S. C. (1956), Brit. med. F., ii, 1267.

TRUELOVE, S. C. (1957), Ibid., i, 1437.

TRUELOVE, S. C. (1958), Ibid., ii, 1072.

TRUELOVE, S. C., and MORRIS-OWEN, R. M. (1958), Itbid., i, 603 .

TRUELOVE, S. C., and WITTS, L. J. (1954), Ibid., ii, 375.

TRUELOVE, S. C., and WITTS, L. J. (1955), Ibid., ii, I041.

TRUELOVE, S. C., and WITTS, L. J. (1959), Ibid., in press.

TULIN, M., KERN, F., Jnr., and ALMY, T. P. (1952), Y. Amer. med. Ass., 150, 559.

WATKINSON, G. (1957), Brit. med. F., ii, 1077. 